\title{
Increasing plasma potassium with amiloride shortens the QT interval and reduces ventricular extrasystoles but does not change endothelial function or heart rate variability in chronic heart failure
}

\author{
C A J Farquharson, A D Struthers
}

Heart 2002;88:475-480

See end of article for authors' affiliations

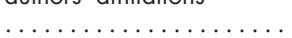

Correspondence to: Professor A D Struthers, University Department of Clinical Pharmacology and Therapeutics, Ninewells Hospital and Medical School, Dundee DDI 9SY, UK;

a.d.struthers@dundee.ac.uk

Accepted 4 July 2002
Objectives: To test whether simply increasing plasma potassium with amiloride would exert any of the same beneficial effects on "surrogate outcome measures" that are seen with spironolactone. The latter has been shown to improve mortality in chronic heart failure, possibly as a result of improvements in endothelial dysfunction, vascular angiotensin converting enzyme (ACE), autonomic function, myocardial fibrosis, ventricular arrhythmias, and QT interval indices.

Design: Randomised, placebo controlled trial.

Setting: Teaching hospital.

Patients and interventions: Double blind crossover study involving 10 patients with New York Heart Association functional class II-III chronic heart failure comparing $5 \mathrm{mg} /$ day amiloride (one month) with placebo.

Main outcome measures: Endothelial function, vascular ACE, collagen markers, 24 hour ECG, and QT interval results.

Results: The amiloride induced increase in serum potassium $(0.4 \mathrm{mmol} / \mathrm{l})$ did not significantly change endothelial dysfunction, vascular ACE, collagen markers, or heart rate variability. However, amiloride significantly improved QT interval indices, reducing both QT dispersion (from $65.7 \mathrm{~ms}$ to $50.9 \mathrm{~ms}$, $p=0.001$ ) and mean maximal corrected QT (from $445 \mathrm{~ms}$ to $435 \mathrm{~ms}, \mathrm{p}=0.008$ ). Amiloride also reduced ventricular extrasystoles $(p<0.05)$.

Conclusions: Amiloride shortens QT interval length and reduces ventricular extrasystoles in chronic heart failure, implying that this effect is caused by potassium retention per se. However, unlike spironolactone, amiloride did not improve endothelial dysfunction, vascular ACE, heart rate variability, or myocardial fibrosis, implying that spironolactone improves these latter effects by aldosterone blockade rather than by simply increasing serum potassium. Therefore, amiloride has fewer beneficial mechanistic effects than spironolactone, but it does share with spironolactone the ability to shorten the QT interval and reduce ventricular extrasystoles.
R ecently RALES (randomized aldactone evaluation study) showed a significant $30 \%$ survival advantage in patients with chronic heart failure (CHF) receiving spironolactone compared with placebo. ${ }^{1}$ Mechanisms that may contribute to this mortality benefit in CHF include spironolactone's ability to improve endothelial dysfunction, to suppress vascular angiotensin converting enzyme (ACE), to reduce myocardial fibrosis, to improve heart rate variability (HRV), to reduce QT interval length and dispersion, and to reduce ventricular extrasystoles. $^{2-5}$

Interestingly, a recently published retrospective analysis of SOLVD (studies of left ventricular dysfunction) focusing specifically on diuretic use showed that potassium losing diuretics were associated with increased risk of arrhythmic death but potassium sparing diuretics were not. ${ }^{6}$ We recently also found a beneficial effect of potassium sparing diuretic use on mortality in patients with CHF. ${ }^{7}$ Amiloride, triamterene, and spironolactone are all potassium sparers but spironolactone has the added effect of blocking aldosterone. In both of the above papers, ${ }^{67}$ the authors did not differentiate between potassium sparing agents; therefore it is difficult to know whether this beneficial effect was indeed caused by increasing potassium per se or whether aldosterone blockade contributed.

We therefore set out to ascertain whether spironolactone's already described effects on these surrogate outcome measures are also found after treatment with a potassium sparing diuretic that does not have aldosterone blocking properties, such as amiloride. We therefore investigated whether amiloride had any effects on endothelial function and vascular ACE activity in CHF patients. We also studied whether amiloride improved HRV as assessed by time and frequency domain (power spectral) HRV analyses. The impact of amiloride on ventricular arrhythmias and QT interval length was also elucidated. ${ }^{589}$ Finally, the effect of amiloride on myocardial fibrotic change was ascertained by using serum measurements of procollagen type III amino terminal peptide (PIIINP), which reflects collagen turnover and is an accepted surrogate marker for myocardial fibrosis. ${ }^{210}$

\section{METHODS}

\section{Study population}

Ten male patients with stable mild to moderate CHF secondary to ischaemic cardiomyopathy (table 1) established on loop

Abbreviations: $\mathrm{ACE}$, angiotensin converting enzyme; $\mathrm{CHF}$, chronic heart failure; HOPE, heart outcomes prevention evaluation; HRV, heart rate variability; L-NMMA, $N^{G}$-monomethyl-L-arginine; $N O$, nitric oxide; NYHA, New York Heart Association; PIIINP, procollagen type III amino terminal peptide; QTc, corrected QT interval; QTcd, corrected QT interval dispersion; QTd, QT interval dispersion; RALES, randomized aldactone evaluation study; SOLVD, studies of left ventricular dysfunction; UK-HEART, United Kingdom heart failure evaluation and assessment of risk trial 
Table 1 Baseline demographic, haemodynamic, humoral, and treatment characteristics of patients

\begin{tabular}{|c|c|}
\hline Parameter & $\begin{array}{l}\text { Value (mean } \\
\text { (SD) or } \\
\text { numbers) }\end{array}$ \\
\hline Age (years) & $69.5(5.6)$ \\
\hline NYHA class II/III & $5 / 5$ \\
\hline Previous/never smokers & $8 / 2$ \\
\hline Average smoking duration (pack years) & $23.5(15)$ \\
\hline Systolic blood pressure $(\mathrm{mm} \mathrm{Hg})$ & $131(7)$ \\
\hline Diastolic blood pressure $(\mathrm{mm} \mathrm{Hg})$ & $77(5)$ \\
\hline Left ventricular ejection fraction (\%) & $28.0(6.9)$ \\
\hline Left ventricular fractional shortening (\%) & $19.5(7.6)$ \\
\hline Serum urea $(\mathrm{mmol} / \mathrm{l})$ & $7.2(1.6)$ \\
\hline Serum creatinine ( $\mu \mathrm{mol} / \mathrm{l})$ & $118(21)$ \\
\hline Serum total cholesterol (mmol/l) & $5.1(0.2)$ \\
\hline Serum urate $(\mathrm{mmol} / \mathrm{l})$ & $0.45(0.8)$ \\
\hline \multicolumn{2}{|l|}{ Mean dose of ACE inhibitor (mg/day) } \\
\hline Lisinopril (7 patients) & $12.9(5.7)$ \\
\hline Enalapril (3 patients) & $16.7(5.7)$ \\
\hline Duration of ACE inhibition (years) & $6.1(3.2)$ \\
\hline Baseline serum ACE activity (taking ACE inhibitor (IU/I)) & $7.8(1.8)$ \\
\hline Random plasma glucose (mmol/l) & $5.7(0.7)$ \\
\hline Daily furosemide dose $40 \mathrm{mg} / 80 \mathrm{mg}$ & $9 / 1$ \\
\hline Daily aspirin dose $75 \mathrm{mg} / 150 \mathrm{mg}$ & $6 / 4$ \\
\hline \multicolumn{2}{|l|}{ Concomitant medication } \\
\hline Nitrates & 5 \\
\hline$\beta$ Blockers & 6 \\
\hline Calcium channel blockers & 4 \\
\hline Statins/antioxidant vitamins & 0 \\
\hline
\end{tabular}

diuretic and ACE inhibitor treatment gave written informed consent to participate in the study, which had prior approval by the Tayside committee on medical research ethics. The study conformed with the principles outlined in the Declaration of Helsinki.

\section{Study experimental design}

One month of treatment with amiloride $5 \mathrm{mg}$ /day was studied using a randomised, placebo controlled, double blind, crossover trial with a two week washout period between treatment phases. Other cardiovascular medications remained constant. Each subject attended for two studies of forearm vascular function, performed at the end of the placebo and amiloride treatment phases. The protocol for these visits is detailed below. At baseline and during the final day of each treatment phase, a 12 lead resting ECG was obtained for subsequent analysis of QT indices, as well as a 24 hour ambulatory ECG monitor to assess HRV and ventricular extrasystoles.

\section{Vascular function protocol}

Following overnight fasting, subjects attended a temperature controlled laboratory $\left(24-26^{\circ} \mathrm{C}\right)$ in our research unit at 8 am and were asked to lie supine. All cardiovascular medications were taken immediately before the start of the study visit. After 20 minutes' rest, the non-dominant brachial artery was cannulated under local anaesthesia with a 27 gauge steel needle mounted on to a 16 gauge epidural catheter. After 30 minutes of saline infusion, baseline forearm blood flow was measured using forearm venous occlusion plethysmography, which has been described by our group in detail previously. ${ }^{511}$

Drugs according to the following study infusion protocol were then infused into the study arm using a constant rate infuser. Firstly, acetylcholine was infused at 25, 50, and $100 \mathrm{nmol} / \mathrm{min}$, each for five minutes, to produce a cumulative dose-response curve. This was followed by sodium nitroprusside at $4.2,12.6$, and $37.8 \mathrm{nmol} / \mathrm{min}$, each for five minutes, and then $N^{\mathrm{G}}$-monomethyl-L-arginine (L-NMMA) at 1, 2, and $4 \mu \mathrm{mol} / \mathrm{min}$ for five minutes each. This in turn was followed by angiotensin I at 64,256 , and $1024 \mathrm{pmol} / \mathrm{min}$ for seven minutes each, and finally angiotensin II was infused at 16, 64, and $256 \mathrm{pmol} / \mathrm{min}$ for seven minutes each.

Between different drugs, the drug infusion set was flushed with saline and sufficient time was allowed for the forearm blood flow to return to baseline values (approximately 20-30 minutes). Forearm blood flow was measured at each baseline and then during the last two minutes of each drug infusion. Blood pressure was measured in the non-infused (control) arm at the beginning of the study, after each saline washout period, and at the conclusion of the study.

Acetylcholine is an endothelium dependent vasodilator and sodium nitroprusside is an endothelium independent vasodilator. L-NMMA is a competitive nitric oxide (NO) synthase inhibitor. Angiotensin I only exerts its vasoconstrictive effect in our forearm model through conversion in the vasculature to angiotensin II, and therefore the vasoconstriction elicited reflects vascular angiotensin I to angiotensin II conversion. ${ }^{12}$

\section{Analysis of QT interval indices}

QT intervals were analysed on resting 12 lead ECGs recorded at baseline and at the end of each treatment phase. Tracings were taken at a speed of $25 \mathrm{~mm} / \mathrm{s}$, with three complexes in each lead used. Only ECGs with more than eight viable leads for analysis were used. The tracings were numbered randomly by a second blinded investigator and analysed blindly by a single investigator (CAJF) using a digitising pad and dedicated QT analysis software (University of Dundee).

The QT interval was taken from the QRS complex to the end of the $\mathrm{T}$ wave (that is, return to the T/P baseline). If $\mathrm{U}$ waves were present, the QT was measured to the nadir of the curve between the $\mathrm{U}$ and $\mathrm{T}$ waves. QT intervals were corrected for rate using Bazett's formula ( QTc $=$ QT/RR $\left.{ }^{\prime} / 2\right)$. QT dispersion (QTd) was defined as the difference between the maximum and minimum QT intervals (QTd = QTmax - QTmin), with corrected QT dispersion (QTcd) being the respective difference in maximum and minimum QTc intervals (QTcd $=$ QTcmax - QTcmin).

\section{Ambulatory 24 hour ECG monitoring}

Twenty four hour ambulatory ECG recordings were obtained for analysis using a standard two channel (four lead) Tracker 2 analogue tape recorder (Reynolds Medical Limited, Hertford, UK), recording standard leads CMI and CM5. This was carried out at baseline and at the end of each course of tablets (the day after the vascular function assessment). Arrhythmias were analysed semiautomatically with the Pathfinder 500 Series analyser system (version 4.63 software, Reynolds Medical Ltd). Also, each recording was individually checked, with RR intervals and QRS configuration manually edited, to ensure correct arrhythmia recognition and classification.

\section{HRV analysis}

HRV was assessed in both time and frequency domains according to published guidelines. ${ }^{13}$ Following visual editing and correct identification of aberrant beats, the RR interval variability on the ambulatory recordings was automatically analysed statistically as a function of time (time domain analysis) by the Pathfinder software. The following time domain indices were evaluated from each 24 hour ECG recording: standard deviation of all RR intervals, 24 hour triangular index, standard deviation of five minute mean RR intervals, and the root mean square of differences of successive RR intervals.

Frequency domain (power spectral) analysis of the RR interval variability was undertaken using fast Fourier transformation, operating on sampled five minute data segments of each hour over the 24 hour recording. Time periods with excessive movement artefact observed were excluded from analysis. Spectral plots were used to identify the low frequency component $(0.03-0.14 \mathrm{~Hz})$ and the high frequency component $(0.18-0.40 \mathrm{~Hz})$. These indices were expressed in 


\begin{tabular}{|c|c|c|}
\hline & Placebo & Amiloride \\
\hline Serum potassium (mmol/l) & $4.4(0.4)$ & $4.8(0.5)^{*}$ \\
\hline Serum urea $(\mathrm{mmol} / \mathrm{l})$ & $6.4(1.5)$ & $7.9(1.7)$ \\
\hline Serum creatinine $(\mu \mathrm{mol} / \mathrm{l})$ & $103(23)$ & $111(26)$ \\
\hline Baseline heart rate (beats $/ \mathrm{min}$ ) & $73(8)$ & $73(7)$ \\
\hline \multicolumn{3}{|c|}{ Baseline blood pressure $(\mathrm{mm} \mathrm{Hg})$} \\
\hline SBP & $134(6)$ & $133(4)$ \\
\hline DBP & $(73) 7$ & $73(8)$ \\
\hline MAP & $93(5)$ & $93(5)$ \\
\hline \multicolumn{3}{|c|}{ Acetylcholine blood pressure $(\mathrm{mm} \mathrm{Hg})$} \\
\hline SBP & $134(6)$ & $134(4)$ \\
\hline DBP & $75(5)$ & $73(4)$ \\
\hline MAP & $94(4)$ & $93(2)$ \\
\hline \multicolumn{3}{|c|}{ Sodium nitroprusside blood pressure $(\mathrm{mm} \mathrm{Hg})$} \\
\hline SBP & $133(8)$ & $133(4)$ \\
\hline DBP & $77(4)$ & $74(4)$ \\
\hline MAP & $95(4)$ & $94(3)$ \\
\hline \multicolumn{3}{|c|}{$N^{\circ}$-monomethyl-L-arginine blood pressure $(\mathrm{mm} \mathrm{Hg})$} \\
\hline SBP & $132(7)$ & $132(6)$ \\
\hline DBP & $75(6)$ & $73(4)$ \\
\hline MAP & $93(5)$ & $93(2)$ \\
\hline \multicolumn{3}{|c|}{ Angiotensin I blood pressure $(\mathrm{mm} \mathrm{Hg})$} \\
\hline SBP & $134(8)$ & $133(5)$ \\
\hline DBP & $75(3)$ & $72(5)$ \\
\hline MAP & $95(3)$ & $93(4)$ \\
\hline \multicolumn{3}{|c|}{ Angiotensin II blood pressure $(\mathrm{mm} \mathrm{Hg})$} \\
\hline SBP & $132(8)$ & $131(8)$ \\
\hline DBP & $73(4)$ & $73(5)$ \\
\hline MAP & $94(3)$ & $93(4)$ \\
\hline
\end{tabular}

${ }^{*} p<0.05$ for difference between treatments.

DBP, diastolic blood pressure; MAP, mean arterial pressure; SBP, systolic blood pressure.

normalised units-that is, the relative percentage of each compared with the total oscillatory power. Separate analyses were undertaken for daytime ( $10 \mathrm{am}$ to $11 \mathrm{pm})$, night time ( $11 \mathrm{pm}$ to $6 \mathrm{am}$ ), and dawn (6 am to $10 \mathrm{am}$ ) hours, with all analyses carried out by a blinded single observer (CAJF).

\section{PIIINP assay}

The effect of treatment on myocardial fibrosis during each study phase was assessed from serum PIIINP measured with a standard commercial radioimmunoassay. ${ }^{14}$

\section{Statistical analysis}

Forearm blood flow rates obtained in the vascular function protocols were expressed as $\mathrm{ml} / \mathrm{min} / 100 \mathrm{ml}$ forearm volume. These forearm blood flows were converted to the ratio between the increase in blood flow in the infused arm and the blood flow in the control arm, expressed as the percentage change in forearm blood flow from the baseline immediately preceding each drug administration (mean (SEM)), calculated according to the method of Whitney. ${ }^{15}$

Clinical characteristics of the placebo and amiloride study visits were compared using Student's paired $t$ tests. Statistical analysis of forearm blood flow measurements, QTd indices, and HRV analysis for individual subjects were compared between treatments using two way analysis of variance with repeated measures, correcting for multiple comparisons for within group effects. A probability value of $p<0.05$ was considered significant and $\mathrm{p}<0.01$ highly significant.

\section{RESULTS}

\section{Subject characteristics}

Baseline forearm blood flow observed in the amiloride treatment group was virtually identical to that of the placebo group $(2.9(0.3) \mathrm{ml} / \mathrm{min} / 100 \mathrm{ml}$ with placebo $v 2.8(0.3) \mathrm{ml} /$ $\mathrm{min} / 100 \mathrm{ml}$ with amiloride, $\mathrm{p}=0.67)$. The baseline forearm blood flow rates preceding each drug infusion were not different between the limbs, indicative of adequate drug washout between each infusion phase.
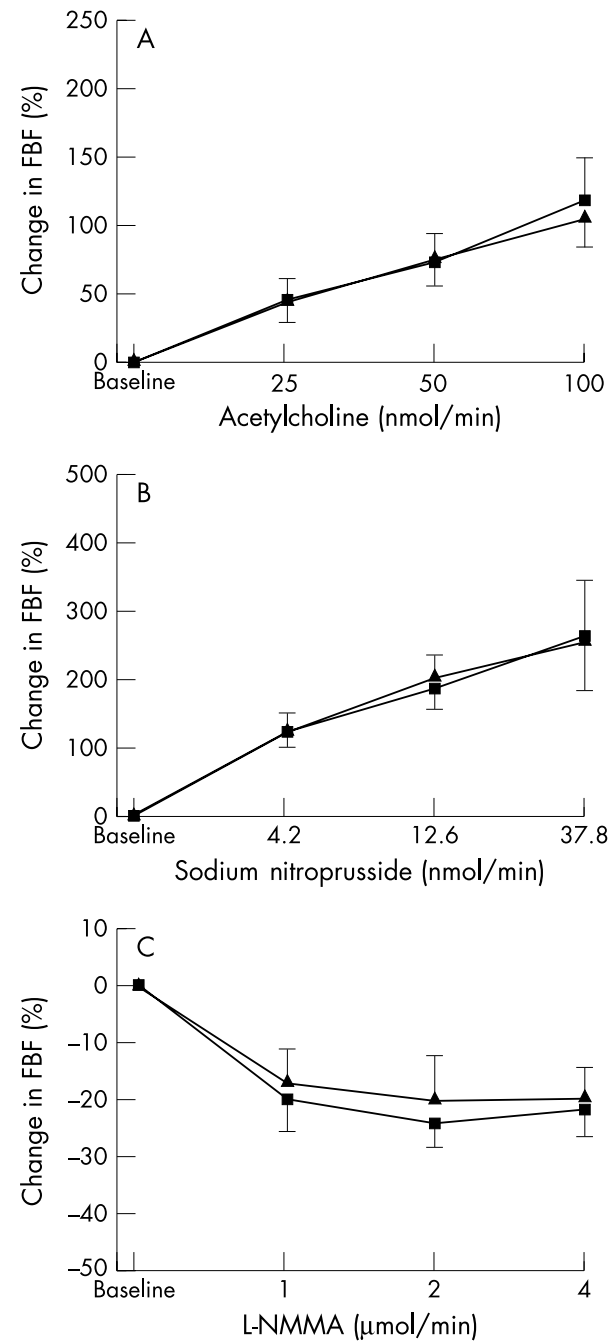

Figure 1 Forearm blood flow responses to three doses of $(A)$ acetylcholine, (B) sodium nitroprusside, and (C) $N^{G}$-monomethyl--arginine (L-NMMA) after placebo (squares) or amiloride (triangles) treatment for one month. Values are mean (SEM).

There was also no significant difference between blood pressure and heart rate either between or during each study day (table 2). Table 2 also shows urea, creatinine, and plasma potassium assay results. There was no perceived subjective or objective change in New York Heart Association (NYHA) functional class with regard to patient symptoms between the two treatment periods.

\section{Forearm vascular blood flow responses}

No significant improvement was seen in endothelial dependent vasodilation in the amiloride treatment group in response to acetylcholine $(\mathrm{p}=0.85$ for difference between whole doseresponse curves) (fig 1 ). No changes were seen in the vasoconstrictive responses to L-NMMA ( $p=0.52)$, implying that tonic NO bioactivity was not changed by amiloride. Amiloride also had no effect on sodium nitroprusside responses $(p=0.94)$.

With regard to the vascular ACE axis, there were no significant differences between the treatment groups in the forearm responses to either angiotensin I $(p=0.81$ for difference between dose-response curves) (fig 2 ) or the control vasoconstrictor angiotensin II $(p=0.90)$.

\section{Changes in QT interval indices}

Amiloride significantly reduced both QTd and QTcd ( $p<0.001$ between baseline and amiloride, $p<0.05$ between 

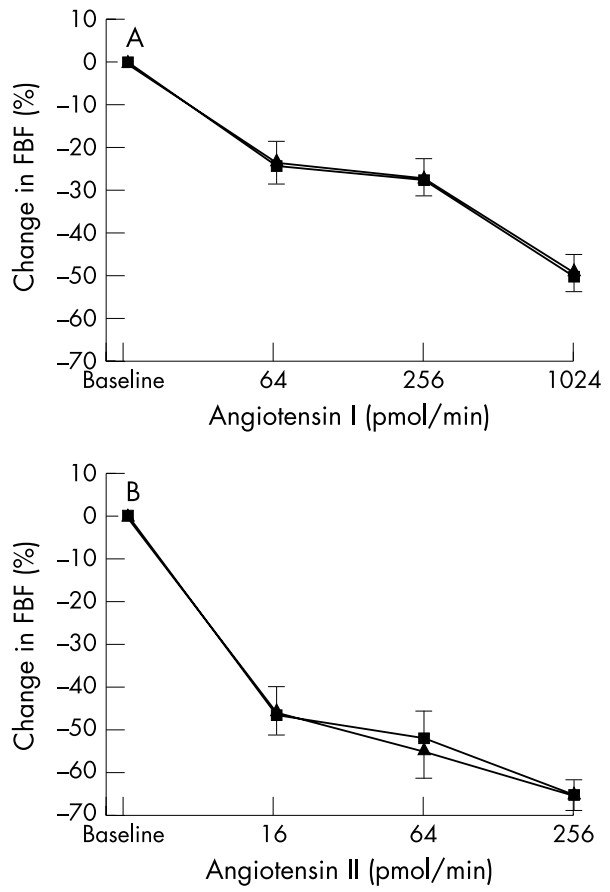

Figure 2 Forearm blood flow responses to three doses of (A) angiotensin I and (B) angiotensin II after placebo (squares) or amiloride (triangles) treatment for one month. Values are mean (SEM).

placebo and amiloride) (table 3). QTcmax was also significantly improved with amiloride $(\mathrm{p}<0.05)$.

\section{HRV analysis}

There were no significant differences from baseline in any of the HRV indices between placebo and amiloride treatment. Neither time domain nor frequency domain measures were significantly affected by active treatment either over the total 24 hour period or during the daytime, night time, or dawn periods (tables 4 and 5).

With regard to arrhythmic activity, there was a significant reduction in the number of ventricular extrasystoles from baseline observed over the 24 hour monitoring period with amiloride compared with placebo (change in ventricular extrasystoles from baseline -14 (279) with placebo $v-310$ (436) with amiloride, $p<0.05)$. No episodes of non-sustained ventricular tachycardia were observed on any of the 30 24-hour tapes.

\section{Changes in serum PIIINP}

There was no significant change in serum concentrations of PIIINP in the amiloride treatment group as compared with either baseline or placebo (table 4).

\section{Results summary}

Table 6 summarises the comparison that we make here with amiloride versus our previous work with spironolactone. ${ }^{35}$

\section{DISCUSSION}

In this study, amiloride caused its expected effect of increasing potassium concentrations. An important point is that the increment we observed here with amiloride is identical in magnitude to the increment we previously saw with spironolactone. ${ }^{5}$

The primary end point of this study was to ascertain whether amiloride, by raising potassium, could improve endothelial dysfunction in CHF. Unlike our previous observations with spironolactone, ${ }^{25}$ a rise in serum potassium of $0.4 \mathrm{mmol} / \mathrm{l}$ induced by amiloride had no effect whatsoever on either basal or stimulated NO activity, nor did amiloride alter vascular ACE inhibition, HRV, or vascular collagen turnover. The only positive effects of amiloride were on QT interval length and ventricular extrasystoles.

Table 3 Changes in QT indices during treatment phases in the 10 patients

\begin{tabular}{lllll}
\hline & Baseline (mean (SD)) & Placebo (mean(SD)) & Amiloride (mean(SD)) & Changes between placebo and amiloride \\
\hline QT dispersion (ms) & $65.4(29.2)$ & $65.7(25.8)$ & $50.9(26.1)^{* *} \dagger$ & $14.8(9.6) 95 \% \mathrm{Cl} 7.9$ to $21.7, \mathrm{p}=0.001$ \\
QTc dispersion (ms) & $68.2(29.0)$ & $68.3(24.8)$ & $52.6(25.3)^{* *} \dagger$ & $15.7(10.0) 95 \% \mathrm{Cl} 8.6$ to $22.9, \mathrm{p}=0.001$ \\
QTcmax (ms) & $442.6(24.6)$ & $445.0(26.6)$ & $435.2(25.7)^{*} \dagger$ & $9.8(9.1) 95 \% \mathrm{Cl} 3.3$ to $16.2, \mathrm{p}=0.008$ \\
\hline
\end{tabular}

${ }^{*} \mathrm{p}<0.05$ between baseline and amiloride treatment phases; ${ }^{*} \mathrm{p}<0.001$ between baseline and amiloride treatment phases; $\dagger p<0.05$ between placebo and amiloride treatment phases.

QTc, corrected QT interval.

Table 4 Effect of amiloride or placebo on mean 24 hour heart rate variability and arrhythmia indices

\begin{tabular}{|c|c|c|c|c|c|}
\hline & Parameter & Baseline & Placebo & Amiloride & p Value* \\
\hline Time domain & RR interval & 929 (149) & $945(137)$ & $947(131)$ & NS \\
\hline \multirow[t]{4}{*}{ Measures } & HRV index & $28(9)$ & $28(9)$ & 31 (10) & NS \\
\hline & SDNN & 114 (39) & $115(36)$ & $114(35)$ & NS \\
\hline & SDANN & $99(32)$ & $97(29)$ & $99(35)$ & NS \\
\hline & RMSSD & $26(6)$ & $26(5)$ & $28(3)$ & NS \\
\hline Frequency domain & LF (n.u.) & $50(22)$ & $56(21)$ & $52(23)$ & NS \\
\hline \multirow[t]{2}{*}{ Measures } & HF (n.u.) & $45(24)$ & $38(22)$ & $42(25)$ & NS \\
\hline & $\mathrm{LF} / \mathrm{HF}$ & $2.9(2.6)$ & $3.0(2.8)$ & $2.7(2.4)$ & NS \\
\hline 24 hour arrhythmia & $\Delta \mathrm{VE} / 24$ hours & NA & $-14(279)$ & $-310(436)$ & $<0.05$ \\
\hline Analysis & NSVT/24 hours & 0 & 0 & 0 & NS \\
\hline Vascular collagen turnover & Serum PIIINP & $3.2(1.0)$ & $3.3(1.3)$ & $3.2(0.8)$ & NS \\
\hline
\end{tabular}

All values are expressed as mean (SD).

* Placebo versus amiloride treatment phases.

$\triangle \mathrm{VE} / 24$ hours, change in frequency of ventricular extrasystoles over 24 hours from baseline; HF, high frequency; LF, low frequency; NSVT, non-sustained ventricular tachycardia; n.u., normalised unit; PIIINP, procollagen type III amino terminal peptide; RMSSD, root mean square of differences of successive RR intervals; SDANN, standard deviation of five minute mean RR intervals; SDNN, standard deviation of all RR intervals. 
Table 5 Diurnal effects of amiloride or placebo on heart rate variability parameters

\begin{tabular}{|c|c|c|c|c|c|}
\hline Time & Parameter & Baseline & Placebo & Amiloride & $\mathrm{p}$ Value* \\
\hline $10 \mathrm{am}$ to $11 \mathrm{pm}$ & $\begin{array}{l}R R(m s) \\
L F \text { (n.u.) } \\
\operatorname{HF} \text { (n.u.) } \\
L F / H F\end{array}$ & $\begin{array}{l}916(149) \\
50(19) \\
47(21) \\
1.9(1.2)\end{array}$ & $\begin{array}{l}938(142) \\
56(19) \\
38(21) \\
2.4(1.5)\end{array}$ & $\begin{array}{l}941(142) \\
53(22) \\
42(24) \\
2.3(1.2)\end{array}$ & $\begin{array}{l}\text { NS } \\
\text { NS } \\
\text { NS } \\
\text { NS }\end{array}$ \\
\hline $11 \mathrm{pm}$ to $6 \mathrm{am}$ & $\begin{array}{l}\text { RR (ms) } \\
\text { LF (n.u.) } \\
\mathrm{HF} \text { (n.u.) } \\
\text { LF/HF }\end{array}$ & $\begin{array}{l}949(118) \\
54(27) \\
42(29) \\
4.8(1.2)\end{array}$ & $\begin{array}{l}968(117) \\
57(25) \\
36(37) \\
4.3(3.5)\end{array}$ & $\begin{array}{l}963(96) \\
55(24) \\
40(26) \\
3.6(2.9)\end{array}$ & $\begin{array}{l}\text { NS } \\
\text { NS } \\
\text { NS } \\
\text { NS }\end{array}$ \\
\hline $6 \mathrm{am}$ to $10 \mathrm{am}$ & $\begin{array}{l}\text { RR (ms) } \\
\text { LF (n.u.) } \\
\text { HF (n.u.) } \\
\text { LF/HF }\end{array}$ & $\begin{array}{l}930(173) \\
50(22) \\
45(22) \\
3.0(2.3)\end{array}$ & $\begin{array}{l}949(161) \\
54(20) \\
45(22) \\
2.4(1.8)\end{array}$ & $\begin{array}{l}958(164) \\
48(23) \\
47(24) \\
1.8(0.9)\end{array}$ & $\begin{array}{l}\text { NS } \\
\text { NS } \\
\text { NS } \\
\text { NS }\end{array}$ \\
\hline
\end{tabular}

Table 6 Comparison of spironolactone versus amiloride in chronic heart failure

\begin{tabular}{lll}
\hline & Spironolactone & Amiloride \\
\hline Endothelial function & Improves $^{4}$ & No effect \\
Vascular ACE & Improves $^{4}$ & No effect \\
Collagen markers & Improves $^{2}$ & No effect \\
Autonomic imbalance & Improves $^{25}$ & No effect \\
QTcmax & Improves $^{5}$ & Improves \\
Ventricular extrasystoles & Improves $^{3}$ & Improves \\
\hline
\end{tabular}

\section{Amiloride and endothelial function}

The potential value of studying endothelial dysfunction has recently been underscored by the findings of four recent studies that endothelial dysfunction is indeed directly associated with future cardiovascular events, including one study using the forearm vessels. ${ }^{17-20}$ Furthermore, there are now three treatments (statins and spironolactone in RALES and ACE inhibitors in HOPE (heart outcomes prevention evaluation)) that in parallel improve brachial artery endothelial dysfunction and reduce cardiovascular events and mortality in large trials. Vitamin E is a fourth treatment with corresponding effects on mortality and on endothelial dysfunction-that is, both are usually neutral. ${ }^{1421}$

Before this study was performed, a number of both animal and human studies had suggested indirectly that changes in potassium concentrations may directly improve endothelial function by the NO pathway. Taddei and colleagues ${ }^{22}$ showed that local intra-arterial infusion of potassium chloride acutely potentiated endothelial dependent vasodilation induced by acetylcholine in hypertensive patients, although not in normotensive subjects. These findings were consistent with previous animal experimental data that high potassium diets improved endothelial dysfunction in the stroke prone spontaneously hypertensive rat $^{23}$ by a mechanism independent of blood pressure reduction alone. Potassium has also been shown to increase the release of endothelium derived relaxing factor from segments of canine femoral artery. ${ }^{24}$ The situation regarding potassium and vascular function has become even more intriguing with the recent hypothesis that potassium may itself be an endothelium derived hyperpolarising factor, ${ }^{25}$ which is also released in response to muscarinic agonists such as acetylcholine. ${ }^{26}$

However, our study data have shown that neither basal NO nor stimulated endothelial function was significantly improved after one month of treatment with amiloride, in contrast to our previous observations with spironolactone in a virtually identically designed study in CHF. ${ }^{5}$ The contrast between the $95 \%$ improvement in endothelial function seen in our earlier study with spironolactone and the $0 \%$ improvement seen here with amiloride is so striking that sample size is unlikely to be a factor, especially since both studies involved the same number of identical subjects. Furthermore, unlike spironolactone, amiloride did not change either angiotensin I or angiotensin II mediated vasoconstriction, implying that amiloride had no effect on vascular ACE inhibition in the presence of chronic ACE inhibitor treatment. Blood pressure, baseline forearm blood flow parameters, and NYHA functional class were unaffected by amiloride, which suggests that haemodynamic changes per se were not responsible for the effects we observed.

\section{Amiloride and QT interval changes}

One of the key observations of this study was the finding that amiloride reduced QTcmax and QTd compared with placebo, as indeed does spironolactone. ${ }^{5}$ Although QTd is controversial in cross sectional studies, ${ }^{78}$ intraindividual changes in the length of the QT interval may be more meaningful, especially when as here they were accompanied by intraindividual decreases in ventricular extrasystole. ${ }^{27}$

It is well recognised that potassium depletion leads to increased QT interval length and to arrhythmogenesis with increased likelihood of development of ventricular dysrhythmia such as torsade de pointes. ${ }^{1628}$ Indeed, in the recent UK-HEART (United Kingdom heart failure evaluation and assessment of risk trial) in patients with CHF, a small reduction in serum potassium was a significant predictor of sudden cardiac death in $\mathrm{CHF}^{29}$ Potassium is one of the main determinants of the QT interval, being responsible for the outward repolarisation currents. Reduction in serum potassium therefore results in slower repolarisation and prolongation of QT intervals. Conversely, intravenous potassium infusion normalises QT prolongation and reduces QTd in patients with CHF.

Therefore, this close relation between potassium and the QT interval is likely to be the main explanation for the beneficial effect that was seen for amiloride on QT intervals. Our data therefore tentatively suggest that amiloride as a potassium sparing diuretic may have indirect antiarrhythmic properties, a concept in keeping with the SOLVD data on diuretics and sudden cardiac death. ${ }^{6}$ Our observations that amiloride produced a significant reduction in ventricular extrasystoles should be treated with caution since ventricular extrasystolic frequency is a far less important marker of arrhythmogenicity than the incidence or duration of non-sustained ventricular tachycardia. Clearly a much greater sample size would be required to observe any effect of amiloride on this more important parameter. 


\section{Amiloride and HRV}

Autonomic dysfunction (as measured by HRV) is a well known independent predictor of mortality in $\mathrm{CHF}^{29}$ Spironolactone has been shown to improve HRV indices in CHF, thought to be modulated by increasing parasympathetic activity. ${ }^{25}$

In this study, amiloride appeared to have no impact whatsoever on either time domain or frequency domain HRV parameters. Our findings show that potassium itself does not modulate autonomic tone in CHF. This is most unlikely to be caused by the small sample size since we have three readings per patient and no trend whatsoever is apparent.

\section{Study limitation}

A limitation of this study is that amiloride and spironolactone were not directly compared in a head to head comparison, which makes comparisons between the two treatments only tentative. Such a direct comparison is now warranted to see whether our current findings can be reproduced.

\section{Conclusions}

This study has shown that in patients with CHF the potassium sparing diuretic amiloride does not improve endothelial dysfunction, modulate vascular ACE inhibition, alter collagen markers, or improve HRV, unlike previous studies with the aldosterone antagonist spironolactone. The implication is that these beneficial effects of spironolactone on these particular surrogate outcome measures occur by a mechanism other than by simply raising serum potassium. However, amiloride did reduce QT interval length and ventricular extrasystoles, suggesting that potassium retention even within the normal range has antiarrhythmic effects in CHF, which may contribute to the reduced sudden death mortality observed with potassium sparing diuretics in the SOLVD trial. Therefore, along with previous results with spironolactone, these results suggest that spironolactone has a wider range of beneficial effects than amiloride on surrogate outcome measures. On the other hand, the reduced QT interval seen with both spironolactone and amiloride may be a contributor, perhaps even a strong contributor, to the mortality reduction seen in RALES.

\section{ACKNOWLEDGEMENTS}

We thank the British Heart Foundation for their invaluable support during this study, as well as Lesley McFarlane and Valerie Godfrey for their expert technical assistance.

\section{Authors' affiliations}

C A J Farquharson, A D Struthers, University Department of Clinical Pharmacology and Therapeutics, Ninewells Hospital and Medical School, Dundee, UK

\section{REFERENCES}

1 Pitt B, Zannad F, Remme WJ, et al, for the Randomized Aldactone Evaluation Study Investigators. The effect of spironolactone on morbidity and mortality in patients with severe heart failure. N Engl J Med 1999;341:709-17.

2 MacFadyen RJ, Barr CS, Struthers AD. Aldosterone blockade reduces vascular collagen turnover, improves heart rate variability and reduces the early morning rise in heart rate in heart failure patients. Cardiovasc Res 1997;35:30-4
3 Barr CS, Lang CC, Hanson J, et al. Effects of adding spironolactone to an ACE inhibitor in CHF secondary to coronary artery disease. Am J Cardiol 1995; 76: 1259-65.

4 Farquharson CAJ, Struthers AD. Spironolactone increases nitric oxide bioactivity, improves endothelial vasodilator dysfunction, and suppresses vascular angiotensin I/angiotensin II conversion in patients with chronic heart failure. Circulation 2000;101:594-97.

5 Yee KM, Pringle SD, Struthers AD. Circadian variation in the effects of aldosterone blockade on heart rate variaiblity and QT dispersion in congestive heart failure. J Am Coll Cardiol 2001;37:1800-7.

6 Cooper HA, Dries DL, Davis CE, et al. Diuretics and risk of arrhythmic death in patients with left ventricular dysfunction. Circulation 1999; 100:1311-5.

7 Struthers AD, Donnan PT, Lindsay P, et al. Effect of allopurinol on mortality and hospitalizations in chronic heart failure: a retrospective cohort study. Heart 2002;87:229-34

8 Barr CS, Naas A, Freeman M, et al. QT dispersion and sudden death in heart failure. Lancet 1994;343:327-9.

9 Darbar D, Luck JF, Davidson NC, et al. Sensitivity and specificity of QTc dispersion for identification of risk of cardiac death in patients with peripheral vascular disease. BM 1996;312:874-9.

10 Klappacher G, Franzen P, Haab D, et al. Measuring extracellular matrix turnover in the serum of patients with idiopathic or ischemic dilated cardiomyopathy and impact on diagnosis and prognosis. Am J Cardiol 1995;75:913-8.

11 Lee AFC, Dick JBC, Bonnar CE, et al. Lisinopril improves arterial function in hyperlipidaemia. Clin Sci 1999;96:441-8.

12 Davidson NC, Barr CS, Struthers AD. C-type natriuretic peptide: an endogenous inhibitor of vascular angiotensin-converting enzyme activity. Circulation 1996:93:1 155-9.

13 Task Force of the European Society of Cardiology and the North American Society of Pacing and Electrophysiology. Heart rate variability: standards of measurement, physiological interpretation, and clinical use. Circulation 1996;93:1043-65

14 Visioli $\mathbf{F}$. Effects of vitamin $\mathrm{E}$ on the endothelium: equivocal? Alpha-tocopherol and endothelial dysfunction. Cardiovasc Res 2001;51:198-201

15 Whitney RJ. The measurement of volume changes in human limbs. J Physiol (Lond) 1953;121:1-27.

16 Gettes LS. Possible role of ionic changes in the appearances of arrhythmias. Pharmacol Ther 1976;2:787.

17 Perticone F, Ceravolo R, Pujia A, et al. Prognostic significance of endothelial dysfunction in hypertensive patients. Circulation 2001;104:191-6.

18 Schachinger V, Britten MB, Zeiher AM. Prognostic impact on adverse longterm outcome of coronary heart disease. Circulation 2000;101:1899-906

19 Al Suwaidi J, Hamasaki S, Higano ST, et al. Long-term follow-up of patients with mild coronary artery disease and endothelial dysfunction. Circulation 2000;101:948-54.

20 Davis SF, Yeung AC, Meredith IT, et al. Early endothelial dysfunction predicts the development of transplant coronary artery disease at 1 year posttransplant. Circulation 1996;93:457-62.

21 Green D, O'Driscoll G, Rankin JM, et al. Lack of effect of vitamin E administration on basal nitric oxide function in male smokers and nonsmokers. Clin Sci 1995;89:343-8.

22 Taddei S, Mattei P, Virdis A, et al. Effect of potassium on vasodilation to acetylcholine in essential hypertension. Hypertension 1994;23:485-90.

23 Sugimoto T, Tobian L, Ganguli MC. High potassium diet protects against dysfunction of endothelial cells in stroke-prone spontaneously hypertensive rats. Hypertension 1988;11:579-85

24 Rubanyi GM, Vanhoutte PM. Potassium-induced release of endothelium-derived relaxing factor from canine femoral arteries. Circ Res 1988:62:1098-103.

25 Edwards G, Dora KA, Gardener M, et al. $\mathrm{K}^{+}$is an endothelium-derived hyperpolarising factor in rat arteries. Nature 1998;396:269-72.

26 Edwards G, Weston AH. Endothelium-derived hyperpolarising factor: a critical appraisal. Prog Drug Res 1998;50:107-33.

27 Pye MP, Cobb SM. Mechanisms of ventricular arrhythmias in cardiac failure and hypertrophy. Cardiovasc Res 1992;26:740-50.

28 Fisch C. Relation of electrolyte disturbances to cardiac arrhythmias. Circulation 1973;47:408

29 Nolan J, Batin PD, Andrews R, et al. Prospective study of heart rate variability and mortality in chronic heart failure. Results of the United Kingdom Heart Failure Evaluation and Assessment of Risk Trial (UK-HEART). Circulation 1998;98:1510-6. 